\title{
Minimax Approximations Subject to a Constraint
}

\author{
By C. T. Fike and P. H. Sterbenz
}

\begin{abstract}
A class of approximation problems is considered in which a continuous, positive function $\varphi(x)$ is approximated by a rational function satisfying some identity. It is proved under certain hypotheses that there is a unique rational approximation satisfying the constraint and yielding minimax relative error and that the corresponding relativeerror function does not have an equal-ripple graph. This approximation is, moreover, just the rational approximation to $\varphi(x)$ yielding minimax logarithmic error. This approximation, in turn, is just a constant multiple of the rational approximation to $\varphi(x)$ yielding minimax relative error but not necessarily satisfying the constraint.
\end{abstract}

1. Introduction. Various authors have investigated approximation problems in which the approximation $f(x)$ is required to satisfy some functional constraint. For example, Cody and Ralston [1] investigated the problem of finding a rational function $f(x)$ with numerator and denominator of degree $N$ such that $f(x)$ satisfies the constraint

$$
f(x)=1 / f(-x)
$$

and minimizes the maximum relative error

$$
\max _{[-\alpha, \alpha]}\left|\frac{f(x)-e^{x}}{e^{x}}\right| .
$$

In this paper, we consider a class of approximation problems including the CodyRalston problem and similar problems that have arisen in other contexts. We show that for a problem in this class there is a unique approximation optimal in the sense that it yields minimax relative error, and we characterize this solution.

2. Relative and Logarithmic Error. Suppose that we want to find a polynomial or rational approximation for a function $\varphi(x)$ on an interval $I: a \leqq x \leqq b$, where $\varphi(x)$ is continuous and does not vanish in $I$. Then, we may assume that $\varphi(x)$ is positive for $x$ in $I$.

Let $V$ be a set of admissible functions. Here $V$ will be either the set of all polynomials of degree $\leqq M$ or else the set $V$ will be the set of all rational functions $p(x) / q(x)$ where $p(x)$ and $q(x)$ are relatively prime polynomials of degree $\leqq M$ and $\leqq N$, respectively, and $q(x)$ does not vanish for $x$ in $I$. We shall refer to such functions $p(x) / q(x)$ as $(M, N)$ rational functions.

For $f(x)$ in $V$, we set

$$
R(x)=\frac{f(x)-\varphi(x)}{\varphi(x)}
$$

Received November 25, 1969, revised November 2, 1970.

AMS 1969 subject classifications. Primary 4115, 4117, 4140; Secondary 6520, 6525.

Key words and phrases. Rational approximation, polynomial approximation, best approximation, constrained approximation, exponential function, starting approximation for square root.

Copyright @ 1971, American Mathematical Society 
and let $\mu$ denote the maximum of $|R(x)|$ for $x$ in $I$. There is a unique function $f^{*}(x)$ in $V$ which minimizes $\mu$ for all $f(x)$ in $V$. We let $\mu^{*}$ denote the value of $\mu$ for $f^{*}(x)$.

Let $W$ be the set of all $f(x)$ in $V$ for which $f(x)>0$ for all $x$ in $I$. Let $c$ be the minimum of $\varphi(x)$ for $x$ in $I$. Then the function $f(x)=c / 2$ is in $W$, and for this function we have $\mu<1$. But any function which is in $V-W$ will yield $\mu \geqq 1$, so $f^{*}(x)$ is in $W$.

For $f(x)$ in $W$, we may consider the logarithmic error

$$
\delta(x)=\log _{e} \frac{f(x)}{\varphi(x)} .
$$

We shall use $\lambda$ to designate the maximum of $|\delta(x)|$ for $x$ in $I$. Thus, with any function $f(x)$, we associate values of $\lambda$ and $\mu$. Clearly,

$$
R(x)=e^{\delta(x)}-1 .
$$

Instead of trying to find $f^{*}(x)$, it is sometimes convenient to try to find a function $f(x)$ in $W$ which minimizes $\lambda$.

In [2], we proved the following theorem for the special case in which $\varphi(x)=\sqrt{ } x$. However, the proof given there is valid for any positive continuous function $\varphi(x)$, so it will not be repeated here.

THEOREM 1. There is a unique function $\bar{f}(x)$ in $W$ which minimizes the maximum of $|\delta(x)|$ on I for all $f(x)$ in $W$. If $\bar{\lambda}$ is the value of $\lambda$ for $\bar{f}(x)$, we have

$$
\bar{\lambda}=\operatorname{arctanh} \mu^{*} \text {. }
$$

$\bar{f}(x)$ is characterized by the fact that it produces an equal-ripple $\delta(x)$, and it is related to $f^{*}(x)$ by

$$
\begin{aligned}
\bar{f}(x) & =f^{*}(x) /\left(1-\left(\mu^{*}\right)^{2}\right)^{1 / 2}, \\
f^{*}(x) & =\bar{f}(x) / \cosh \bar{\lambda}
\end{aligned}
$$

3. Constraints. In addition to the two related problems of finding $f^{*}(x)$ and $\bar{f}(x)$, there are some cases in which it is desirable to consider a third problem in which $f(x)$ is required to satisfy an identity satisfied by $\varphi(x)$. Three examples are:

(1) Find the best $(N, N)$ rational approximation $f(x)$ for $e^{x}$ on $-\alpha \leqq x \leqq \alpha$ such that $f(-x)=1 / f(x)$.

(2) For $0<\alpha<1$, find the best $(N, N)$ rational approximation $f(x)$ for $\sqrt{ } x$ on $\alpha \leqq x \leqq 1 / \alpha$ such that $f(1 / x)=1 / f(x)$.

(3) For $N>0$ and $0<\alpha<1$, find the best $(N+1, N)$ rational approximation $f(x)$ for $\sqrt{ } x$ on $\alpha \leqq x \leqq 1 / \alpha$ such that $x f(1 / x)=f(x)$.

In each case, by the best approximation, we mean the one which minimizes $\mu$ subject to the constraint. An approximation of the first type is found by Cody and Ralston in [1] and by Kahan in [3]. Maehly studied an approximation of the second type. See the appendix of [4]. In [4], Cody finds an approximation of the third type. These constraints often simplify the problem of finding the best approximation by reducing the number of coefficients.

In each case, we have a constraint $C$. Let $U$ be the set of all functions $f(x)$ in $V$ which satisfy the constraint $C$. We shall require that the set $U$ have the following properties:

(a) $\bar{f}(x)$ is in $U$. 
(b) If $f(x)$ is in $U \cap W$, then for any $x$ in $I$ there is a point $y$ in $I$ such that $\delta(y)=-\delta(x)$.

(c) For any $f(x)$ in $U-W$ there is a $g(x)$ in $U \cap W$ which has a smaller $\mu$ than $f(x)$ does.

We first show that for each of the three examples considered above, $U$ satisfies these properties. That $\bar{f}(x)$ is in $U$ follows from the uniqueness of $\bar{f}(x)$, since otherwise we would have another function in $W$ with the same value of $\lambda$, namely $1 / f(-x)$ in (1), $f(1 / x)$ in (2), and $x f(1 / x)$ in (3). For property (b) of $U$, we use $y=-x$ in (1) and $y=1 / x$ in (2) and (3). For property (c) of $U$, we first observe that our definition of $V$ implies that every function $f(x)$ in $V$ is bounded on $I$. For examples (1) and (2), this implies that $f(x)$ cannot vanish in the interval $I$, so if $f(x)$ is in $U-W$, we take $g(x)=-f(x)$. In the third example, we may always take $g(x)=\epsilon+\epsilon x$, where $\epsilon$ is a small positive constant such that the maximum of $g(x)$ is less than the minimum of $\varphi(x)$ for $x$ in $I$.

We now address the problem of finding $f(x)$ in $U$ which minimizes $\mu$. Because of property (c), we need consider only functions in $U \cap W$. But for any function $f(x)$ in $U \cap W$, we have, by (1), $e^{\lambda}-1 \geqq R(x) \geqq e^{-\lambda}-1$, and since $\delta(x)$ is continuous on $I$ there is a point $x$ in $I$ with $|\delta(x)|=\lambda$. But by property (b), there is a point $y$ in $I$ with $\delta(y)=-\delta(x)$, so $R(x)$ assumes both the values $e^{\lambda}-1$ and $e^{-\lambda}-1$ in $I$. Then, for $f(x)$ we have

$$
\mu=e^{\lambda}-1 .
$$

Since $\bar{f}(x)$ minimizes $\lambda$ for all $f(x)$ in $W$, we have $\lambda \geqq \bar{\lambda}$, and therefore (2) implies $\mu \geqq e^{\bar{\lambda}}-1$. By property (a), $\bar{f}(x)$ is in $U \cap W$. Then, using $\bar{\mu}$ to denote the value of $\mu$ for $\bar{f}(x)$, we have, from (2), $\bar{\mu}=e^{\bar{\lambda}}-1$. Then, $\bar{f}(x)$ minimizes $\mu$ for all $f(x)$ in $U$. If $g(x)$ is any function in $U$ with

$$
\mu=e^{\bar{\lambda}}-1,
$$

then (2) and (3) imply that $\lambda=\bar{\lambda}$, so the uniqueness of the function minimizing the maximum of $|\delta(x)|$ implies that $g(x)=\bar{f}(x)$. We have proved:

THEOREM 2. $\bar{f}(x)$ is the unique function in $U$ which minimizes the maximum of $|R(x)|$ for all $f(x)$ in $U$. For $\bar{f}(x)$, we have

$$
e^{-\bar{\lambda}}-1 \leqq \bar{R}(x) \leqq e^{\bar{\lambda}}-1 \text { and } \bar{\mu}=e^{\bar{\lambda}}-1
$$

The relation between the solution $\bar{f}(x)$ of the constrained problem and the solution $f^{*}(x)$ of the unconstrained problem is given in Theorem 1 .

4. Comments. Since $\bar{f}(x)$ produces an equal-ripple $\delta(x)$, it produces an $R(x)$ which has the correct number of alternating sign extrema but which is not equal-ripple because the maximum is larger than the absolute value of the minimum. Thus, with constraints of this sort, the best-fit problem has a solution which does not produce an equal-ripple error curve.

For the first example, approximating $e^{x}$, we would usually select $V$ so that the approximation $f^{*}(x)$ is accurate to better than word length. Since

$$
\left(1-\left(\mu^{*}\right)^{2}\right)^{1 / 2} \approx 1-\frac{1}{2}\left(\mu^{*}\right)^{2},
$$

this means that $f^{*}(x)$ and $\bar{f}(x)$ agree to more than twice word length, and so do 
$e^{\bar{x}}-1$ and $\left|e^{-\bar{x}}-1\right|$. Thus, we will be equally satisfied with either $f^{*}(x)$ or $\bar{f}(x)$. Since the constraint reduces the number of coefficients, it may be easier to consider the constrained problem.

For $\sqrt{ } x$, we usually look for a starting approximation, and then use Newton's method. In this case, $f^{*}(x)$ and $\bar{f}(x)$ may be noticeably different, since the approximation is not very accurate. But we showed in [2] that $\bar{f}(x)$ minimizes the maximum relative error after one or more iterations, so we would prefer to have $\bar{f}(x)$ instead of $f^{*}(x)$. Then the constraint may be used to simplify the computation as in [4].

IBM Systems Research Institute

New York, New York 10017

1. W. J. Cody \& ANTHony Ralston, "A note on computing approximations to the exponential function," Comm. $A C M$, v. 10, 1967, pp. 53-55.

2. P. H. STERBENZ \& C. T. FIKE, "Optimal starting approximations for Newton's method," Math. Comp., v. 23, 1969, pp. 313-318. MR 39 \#6511.

3. W. KAHAN, "Library tape functions EXP, TWOXP, and .XPXP.," Programmers' Reference Manual, University of Toronto, 1966 . (Mimeographed.)

4. W. J. CoDY, "Double-precision square root for the CDC-3600," Comm. $A C M$, v. 7, 1964, pp. 715-718. 